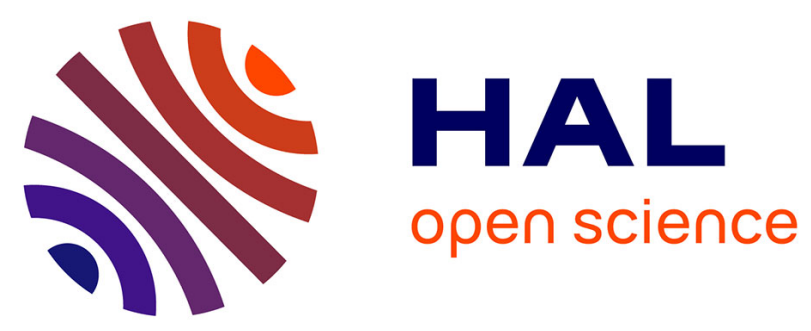

\title{
Activation energies and the extended jump model: How temperature affects reorientation and hydrogen-bond exchange dynamics in water
}

\author{
Zeke A Piskulich, Damien Laage, Ward H Thompson
}

\section{To cite this version:}

Zeke A Piskulich, Damien Laage, Ward H Thompson. Activation energies and the extended jump model: How temperature affects reorientation and hydrogen-bond exchange dynamics in water. Journal of Chemical Physics, 2020, 153 (7), pp.074110. 10.1063/5.0020015 . hal-02952473

\section{HAL Id: hal-02952473 https://hal.sorbonne-universite.fr/hal-02952473}

Submitted on 29 Sep 2020

HAL is a multi-disciplinary open access archive for the deposit and dissemination of scientific research documents, whether they are published or not. The documents may come from teaching and research institutions in France or abroad, or from public or private research centers.
L'archive ouverte pluridisciplinaire HAL, est destinée au dépôt et à la diffusion de documents scientifiques de niveau recherche, publiés ou non, émanant des établissements d'enseignement et de recherche français ou étrangers, des laboratoires publics ou privés. 


\title{
Activation Energies and the Extended Jump Model: How Temperature Affects Reorientation and Hydrogen-Bond Exchange Dynamics in Water
}

\author{
Zeke A. Piskulich, ${ }^{1}$ Damien Laage, ${ }^{2,}{ }^{1}$ and Ward H. Thompson ${ }^{1,2}$
}

1)

Department of Chemistry, University of Kansas, Lawrence, KS 66045, USA

2)PASTEUR, D'epartment de Chimie, Ecole Normale Sup'erieure, PSL University, Sorbonne Universit'e, CNRS,' Paris, 75005,

France

(Dated: 24 September 2020)

Hydrogen-bond exchanges drive many dynamical processes in water and aqueous solutions. The extended jump model (EJM) provides a quantitative description of $\mathrm{OH}$ reorientation in water based on contributions from hydrogen-bond exchanges, or jumps, and the "frame" reorientation of intact hydrogen-bond pairs. Here, we show that the activation energies of $\mathrm{OH}$ reorientation in bulk water can be calculated accurately from the EJM, and that the model provides a consistent picture of hydrogen-bond exchanges based on molecular interactions. Specifically, we use the recently developed fluctuation theory for dynamics to calculate activation energies, from simulations at a single temperature, of the hydrogen-bond jumps and the frame reorientation, including their decompositions into contributions from different interactions. These are shown to be in accord, when interpreted using the EJM, with the corresponding activation energies obtained directly for OH reorientation. Thus, the present results demonstrate that the EJM can be used to describe the temperature dependence of reorientational dynamics and the underlying mechanistic details.

${ }^{1}$ Electronic mail: damien.laage@ens.psl.eu

2 Electronic mail: wthompson@ku.edu 


\section{INTRODUCTION}

In liquid water at ambient conditions, a broad range of dynamical processes have been found to exhibit similar temperature dependences. These include, for example, molecular reorientation measured by $\mathrm{NMR}^{1,2}$ and femtosecond infrared spectroscopies, ${ }^{3-5}$ vibrational frequency dephasing, ${ }^{4-6}$ dielectric relaxation, ${ }^{7}$ viscosity, ${ }^{8-}$ 11 and structural dynamics probed by quasi-elastic neutron scattering. . $2,13^{2}$ The comparable activation energies measured for these processes imply that the associated energy barriers have similar heights and could thus suggest that they share a common ratedetermining elementary step. Hydrogen-bond (H-bond) exchanges are likely this common underlying mechanism, as they have already been shown to be the major reorientation pathway. ${ }^{14}$

However, an important limitation in establishing activation energies in water arises from the markedly nonArrhenius behavior at ambient conditions. ${ }^{8,15-17}$ The traditional determination from a series of measurements at different temperatures is thus ambiguous and sensitively depends on the chosen temperature interval. This issue was recently addressed by a fluctuation theory approach for dynamics, ${ }^{18-24}$ which permits the calculation of activation energies from molecular dynamics simulations at a single temperature. This method further provides important insight in the activation energy components, and has been successfully applied to a broad range of dynamical processes in water.

Here, we use the fluctuation theory method to identify the molecular origin of the reorientation activation energy and its connection to hydrogen-bond exchange dynamics. In contrast to the long-held Debye diffusion picture, water reorientation was shown ${ }^{14}$ to be mostly caused by sudden, large-amplitude angular jumps during hydrogen-bond exchanges, along with the slower tumbling of intact hydrogen-bonds, and the extended jump model (EJM) was proposed to connect this mechanism to the water reorientation time. However, while hydrogenbond jumps have been characterized over a broad temperature range, ${ }^{25}$ the EJM has so far mostly been used at ambient temperature. Our present goal is therefore twofold: 1) we will first assess the EJM's ability to describe the reorientation time temperature dependence and activation energy, and 2) we will use this model to gain a molecular insight in the activation energy's origin as well as its connection with H-bond exchanges.
The remainder of the paper is organized as follows. The details of $\mathrm{OH}$ reorientation and its description within the extended jump model are presented in Sec. II with particular attention on the associated activation energies. The details of the molecular dynamics simulations that are used to determine the timescales and activation energies are given in Sec. III. The $\mathrm{OH}$ reorientation timescales and their activation energies are presented in Sec. IVA. These are compared quantitatively with the corresponding results for the H-bond jump time, the distribution of jump angles, and the frame reorientation time in the remainder of Sec. IV and the fidelity of the EJM is discussed quantitatively. Finally, conclusions are offered in Sec. V.

II. THEORY

\section{A. Water $\mathrm{OH}$ Group Reorientation}

Water reorientational dynamics are frequently described in terms of the time correlation function (TCF),

$$
C_{n}(t)=\mathrm{h} P_{n}\left[\sim e_{O H}(0) \cdot \sim e_{O H}(t)\right] \mathrm{i},
$$

where $P_{n}$ is the $n^{\text {th }}$-order Legendre polynomial and $\sim e_{\mathrm{OH}}(t)$ is the unit vector along the $\mathrm{OH}$ bond undergoing reorientation (Figure 1). The second-order TCF $C_{2}(t)$ is accessible experimentally via polarizationresolved infrared pump-probe measurements, which under most conditions probe the anisotropy decay $r(t)=0.4 C_{2}(t) .26,27$ As we have shown previously, this reorientation has a different temperature dependence than that of the integrated reorientation time, $\left\langle\tau_{2}\right\rangle=\int_{0}^{\infty} C_{n}(t) \mathrm{d} t$, which is typically measured in nuclear magnetic resonance experiments. ${ }^{21}$

It is useful, in examining the mechanism(s) underlying the reorientational dynamics, to compare the behavior for different orders, $n$, of the TCFs and to determine the associated reorientation times. For example, a characteristic feature of systems obeying Debye rotational diffusion is that reorientation times are governed by the rotational diffusion coefficient $D_{R}$,

$$
\tau_{n}^{\text {Debye }}=\frac{1}{n(n+1) D_{R}}
$$

which leads to direct relationships between the reorientational timescales obtained for different orders. That is,

$$
\tau_{1}^{\text {Debye }} / \tau_{2}^{\text {Debye }}=3 \text { and } \tau_{1}^{\text {Debye }} / \tau_{3}^{\text {Debye }}=6 .
$$




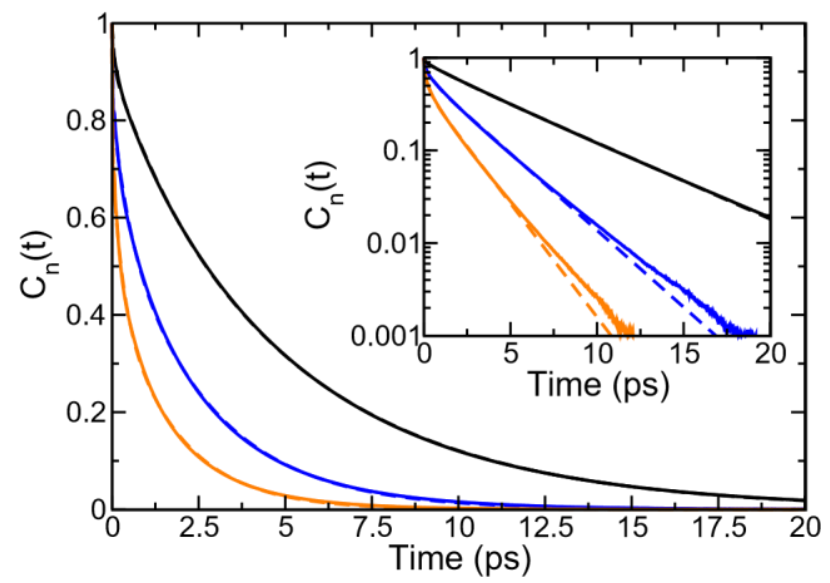

FIG. 1: Water OH group reorientational TCFs, Eq. (1), at $298.15 \mathrm{~K}$ for $n=1$ (black), 2 (blue), and 3 (orange); triexponential fits using Eq. (3) are shown as dashed lines of the same color. Inset shows the results on a semi-log plot.

The $\mathrm{OH}$ reorientational dynamics in water captured in $C_{n}(t)$ exhibit three timescales. The fastest of these (tens of fs) is associated with inertial, or ballistic, motion and is followed by slower dynamics (hundreds of fs) due to librational motion of the $\mathrm{OH}$ group within the intact Hbond. The longest timescale is $2.5-2.6 \mathrm{ps}^{28-30}$ and is determined by $\mathrm{H}$-bond dynamics. Because of this behavior, $C_{n}(t)$ can be fit using a sum of three exponentials,

$$
C_{n}(t)=\sum_{\alpha} A_{\alpha, n} e^{-k_{\alpha, n} t},
$$

where $\alpha=$ inertial, librational, and Hbond. The last is associated with $\mathrm{H}$-bond breaking and making; it is this reorientational dynamics that the EJM describes and which will thus be the focus of the remainder of this paper. For simplicity, we will henceforth omit the "Hbond" and refer to this rate constant as $k_{n}=1 / \tau_{n}$, where $\tau_{n}$ is the corresponding timescale. The $A_{\alpha, n}$ are the amplitudes associated with the three timescales, which for the longest timescale will be referred to as $A_{n}$.

\section{B. Extended Jump Model for Water Reorientation}

It is now well-established that water reorientation is not a rotationally diffusive process, but modifications to the overall picture of the mechanism came in steps. Starting from the Debye diffusion picture, which assumes that reorientation proceeds through a succession of infinitesimally small amplitude angular jumps, Ivanov extended this model to describe reorientation through (uncorrelated) finite amplitude angular jumps, ${ }^{31}$ but did not apply the resulting theory to any molecular system.
The Ivanov model further assumed that the reorientation axis remained fixed and underwent no reorientation between the angular jumps. More recently, Laage and Hynes demonstrated, by analysis of molecular dynamics trajectories, that $\mathrm{OH}$ bonds in liquid water do reorient through finite amplitude jumps associated with the exchange of $\mathrm{H}$-bond acceptors. The average angle spanned in such jumps is around $70^{\circ}$ and this represents the dominant contribution to $\mathrm{OH}$ reorientation, but it is not the sole one. They proposed the extended jump model $^{14,32,33}$ (EJM) that included both the H-bond jumps and an additional contribution associated with the reorientation of the intact $\mathrm{H}$-bond in between $\mathrm{H}$-bond exchanges, which is referred to as "frame" reorientation.

The EJM has been used to describe how $\mathrm{OH}$ reorientation in water is affected by ions ${ }^{33-36}$ as well as hydrophobic ${ }^{37}$ and amphiphilic ${ }^{38}$ solutes. In addition, the EJM has been used to gain insight into water reorientational dynamics at interfaces that range from extended aqueous surfaces 39,40 to biomolecular hydration shells ${ }^{41}$ and confining frameworks including zeolites, ${ }^{42}$ reverse micelles, ${ }^{43}$ and mesoporous silica. ${ }^{44,45}$ The EJM has also been extended to describe $\mathrm{OH}$ reorientation in alcohols.46,47

The EJM describes the longest $\mathrm{OH}$ reorientation timescale as a combination of large-amplitude angular jumps associated with exchange of $\mathrm{H}$-bond acceptor and

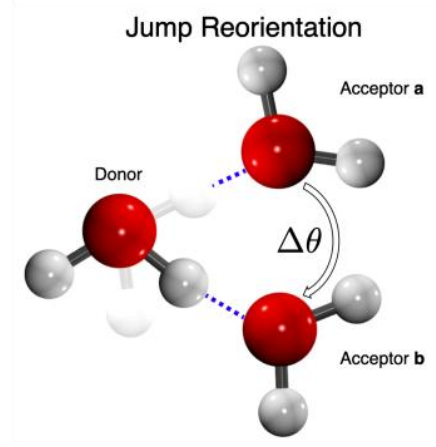

Frame Reorientation

FIG. 2: Schematic illustration of the two components of the extended jump model for $\mathrm{OH}$ reorientation: Largeamplitude angular jumps associated with the exchange of H-bond acceptors (left) and the frame reorientation of the intact $\mathrm{H}$-bond pair between jumps (right).

the frame reorientation of the individual intact $\mathrm{H}$-bond pair, as illustrated in Fig. 2. Mathematically, this gives the $n^{\text {th }}$-order reorientational time, $\tau_{n}$, as

$$
\frac{1}{\tau_{n}}=\frac{1}{\tau_{n}^{\text {jump }}}+\frac{1}{\tau_{n}^{\text {frame }}},
$$


where $\tau_{n}$ jump and $\tau_{n}$ frame are the jump and frame contributions, defined in greater detail below. Equivalently, this can be written in terms of effective rate constants associated with each timescale,

$$
k_{n}=k_{n}^{\text {jump }}+k_{n}^{\text {frame }},
$$

where $k_{n}^{X}=1 / \tau_{n}^{X}$ for each timescale in Eq. (4).

C. Activation Energies and the Fluctuation Theory for Dynamics

The water $\mathrm{OH}$ reorientation activation energy is

$$
E_{a, n}=-\frac{\partial \ln \left(1 / \tau_{n}\right)}{\partial \beta},
$$

where $\beta=1 / k_{B} T$. Within the EJM, $E_{a, n}$ is related to the jump and frame reorientation activation energies via Eq. (5) as

$$
\begin{aligned}
E_{a, n} & =\frac{k_{n}^{\text {jump }}}{k_{n}} E_{a, n}^{\text {jump }}+\frac{k_{n}^{\text {frame }}}{k_{n}} E_{a, n}^{\text {frame }} \\
& =\frac{\tau_{n}}{\tau_{n}^{\text {jump }}} E_{a, n}^{\text {jump }}+\frac{\tau_{n}}{\tau_{n}^{\text {frame }}} E_{a, n}^{\text {frame }}
\end{aligned}
$$

This contrasts with the activation energies expected within the Debye model Eq. (2),

$$
E_{a, n}^{\text {Debye }}=-\frac{\partial \ln D_{R}}{\partial \beta},
$$

which are explicitly independent of the order of the TCF. While the ratios of the reorientation timescales have frequently been used to examine the mechanism of reorientation in liquids,14,28,32,46,48-50 the behavior of $E a, n$ on the order $n$ of the TCF has not previously been explored, nor have the relative contributions of the jump and frame reorientations. The following sections lay out a simple technique for calculating each term in Eq. (7) using fluctuation theory for dynamics and address these issues.

Fluctuation theory for dynamics yields the analytical derivative of a rate constant or timescale with respect to $\beta$ rather than the numerical derivative obtained from an Arrhenius analysis. It is thus particularly advantageous when applied to systems that deviate from the Arrhenius relation, as do many dynamical properties of liquid water. Within this framework, we have previously shown that the derivative of Eq. (1) with respect to $\beta$ can be expressed as a new TCF,

$$
\frac{\partial C_{n}(t)}{\partial \beta}=-\left\langle\delta H(0) P_{n}\left[\vec{e}_{O H}(0) \cdot \vec{e}_{O H}(t)\right]\right\rangle \equiv-C_{n, H}(t),
$$

where $\delta H(0)=H(0)-\mathrm{h} H \mathrm{i}$ is the fluctuation in energy. This TCF, like $C_{n}(t)$ itself, can be evaluated from simulations at a single temperature, avoiding the necessity for an Arrhenius analysis. In other words, Eq. (9) gives the analytical derivative of the TCF with respect to temperature in contrast to the numerical derivative obtained from an Arrhenius plot.

The derivative TCF given in Eq. (9) can be fit by taking the derivative of Eq. (3) with respect to $\beta$,

$$
\frac{\partial C_{n}(t)}{\partial \beta}=\sum_{\alpha}\left[\frac{\partial A_{\alpha, n}}{\partial \beta}-\frac{\partial k_{\alpha, n}}{\partial \beta} A_{\alpha, n} t\right] e^{-k_{\alpha, n} t}
$$

where $A_{\alpha, n}$ and $k_{\alpha, n}$ are now constants calculated from the fit of Eq. (3) and $\partial A_{\alpha, n} / \partial \beta$ and $\partial k_{\alpha, n} / \partial \beta$ are fitting parameters. The latter can be used to determine the activation energy associated with each timescale as $E_{a, n}=$ $-k_{\alpha, n}^{-1}\left(\partial k_{\alpha, n} / \partial \beta\right)$, which is equivalent to Eq. (6). ${ }^{51}$

A key advantage of the fluctuation theory approach is the additional mechanistic insight it provides. Specifically, the total system energy fluctuation can be decomposed into physically meaningful components, which can be used to determine the contributions to the activation energy for each. ${ }^{19,21-24,52}$ For example, in the case of the fixed-charge classical MD simulations used in the present work, it is natural to divide the energy fluctuation as $\delta H(0)=\delta K E(0)+\delta V_{L J}(0)+\delta V_{\text {Coul }}(0),(11)$ where $K E$ is the total kinetic energy and $V_{L J}$ and $V_{\text {Coul }}$ are the total Lennard-Jones and Coulombic potential energies. Using this in Eq. (9) gives a rigorous decomposition of $\partial C_{n}(t) / \partial \beta$ into contributions from these components of the energy. By fitting each of these derivative contributions with a function of the form of Eq. (10), we can obtain the activation energy components

$$
E_{a, n}=E_{a, n}^{K E}+E_{a, n}^{L J}+E_{a, n}^{C o u l} .
$$

Here, for example, $E_{a, n}{ }^{\text {Coul }}$ comes from fitting the TCF in Eq. (9) with $\delta H(0)$ replaced by $\delta V_{\text {Coul }}(0)$.

The interpretation of this result is best understood in the context of Tolman's analysis of the activation energy. ${ }^{22,53-55}$ Tolman showed that the activation energy for a chemical reaction is the average energy of reacting species minus the average energy of reactants,

$E_{a}=\mathrm{h}$ Eireacting $-\mathrm{h}$ Eireactant. In this context, one can view, for example, the kinetic energy component of the activation energy as the average kinetic energy of reacting species minus the average kinetic energy of reactants. In other words, the activation energy components are measures of how energy in different motions or interactions helps (or hinders) passage over the barrier for the process. This is how Eq. (12) can be understood to provide mechanistic information, information which is not available by other methods. This activation energy decomposition gives an even more detailed test of the EJM as will be discussed below. 
D. Jump Reorientation

The contribution to $\mathrm{OH}$ reorientation due to $\mathrm{H}$-bond exchanges involves two factors. The first is the jump time, $\tau_{0}=1 / k_{0}$, which is the inverse rate constant for the "reaction" in which an $\mathrm{OH}$ switches from one $\mathrm{H}$-bond acceptor to another. This timescale, unlike the others discussed in this study, is independent of the order $n$. The second is the effect of the "jump angle," $\Delta \theta$, which is defined as the effective angle traversed (in a lab-fixed frame) by the $\mathrm{OH}$ vector in the process of the exchange of H-bond acceptors. ${ }^{14,32}$ This angle is large, $\sim 70^{\circ}$ on average. The overall effect of the jumps on the reorientational TCF depends on $n$ and is given by

$$
k n j u m p=k 0 \overline{W n} .
$$

Here, $\bar{w}_{n}$ is the average value of the weighting function for the jump angle, which was derived by Ivanov, ${ }^{31}$

$$
w_{n}(\Delta \theta)=1-\frac{\sin [(2 n+1) \Delta \theta / 2]}{(2 n+1) \sin [\Delta \theta / 2]} .
$$

The average value is obtained by averaging over the distribution of jump angles, $P(\Delta \theta)$,

$$
\bar{w}_{n}=\int_{0}^{\pi} w_{n}(\Delta \theta) P(\Delta \theta) d \Delta \theta .
$$

Mathematically, the jump angle can be taken as the $O_{a}$ $\cdots O_{d} \cdots O_{b}$ angle at the jump transition state where $a$ and $b$ indicate the original and new H-bond acceptors, respectively, and $d$ the $\mathrm{H}$-bond donor. In practice it is sufficient to calculate this angle at the first timestep at which a new $\left(O_{d}-H_{d} \cdots O_{b}\right) \mathrm{H}$-bond is formed. Though $w_{n}(\Delta \theta)$ does not depend on temperature, its average value, $w_{n}$, does because it involves the jump angle distribution, $P(\Delta \theta)$.

The jump time $\tau_{0}$, and associated rate constant $k_{0}$, can be calculated using the stable-states picture ${ }^{56}$ for the Hbond exchange process. Specifically, the "side-side" time correlation function,

$$
C_{a b}(t)=\mathrm{h} n_{a}(0) n_{b}(t) \mathrm{i},
$$

is calculated, where $n_{a}\left(n_{b}\right)$ is equal to 1 if the $\mathrm{OH}$ of interest is $\mathrm{H}$-bonded to molecule $a(b)$, and zero otherwise. Absorbing boundary conditions are used such that once an exchange occurs, no further exchanges are considered. The function, $1-C_{a b}(t)$ then decays at longer times with the timescale $\tau_{0}$; in practice, it is fit to a double exponential to account for transient dynamics at early times. ${ }^{57}$ In this work, we have defined H-bonds by strict geometric criteria: $R_{O d-} O_{a} \leq 3.1^{\circ} \mathrm{A}, r_{H d-} O_{a} \leq 2.0^{\circ} \mathrm{A}$, and $\alpha_{H_{d}-O_{d}-O_{a}} \leq 20$.
We have previously shown that the derivative of $1-$ $C_{a b}(t)$ with respect to $\beta$ is given by ${ }^{18}$

$$
\frac{\partial\left[1-C_{a b}(t)\right]}{\partial \beta}=\left\langle\delta H(0) n_{a}(0) n_{b}(t)\right\rangle \equiv C_{a b, H}(t) .
$$

In that work, we demonstrated that the derivative correlation function peaks at a time of $\tau_{0}$ and at a height of $E_{a, 0} / e$ (where $E_{a, 0}$ is the jump time activation energy and $e$ is Euler's number) if the decay is assumed to be single exponential. Here, we adopt the more accurate approach of a double exponential fit for the decay and hence for the derivative TCF in Eq. (17), analogous to Eq. (10), which is then used to determine $\partial k_{0} / \partial \beta$.

Because both $k_{0}$ and $\bar{w}_{n}$ depend on temperature, the derivative of $k_{n}{ }^{j u m p}$ with respect to $\beta$ is

$$
\frac{\partial k_{n}^{j u m p}}{\partial \beta}=\frac{\partial k_{0}}{\partial \beta} \bar{w}_{n}+k_{0} \frac{\partial \bar{w}_{n}}{\partial \beta} .
$$

The jump contribution activation energy can then be calculated by dividing both sides by $k_{n}{ }^{\text {jump }}={\overline{k_{0}}}_{W_{n}}$ and changing the sign, yielding

$$
\begin{aligned}
E_{a, n}^{\text {jump }} & =-\frac{1}{k_{0}} \frac{\partial k_{0}}{\partial \beta}-\frac{1}{\bar{w}_{n}} \frac{\partial \bar{w}_{n}}{\partial \beta} \\
& \equiv E_{a, 0}+E_{a, n}^{\Delta \theta} .
\end{aligned}
$$

Thus, it is the sum of the jump time activation energy, $E_{a, 0}$, (which is not to be confused with the jump reorientation contribution activation energy, $E_{a, n}{ }^{\text {jump }}$, which includes the jump angle temperature dependence) and the activation energy associated with the jump angle weighting, $E_{a, n}^{\Delta \theta}$. Note that only the latter depends on the order $n$ of the TCF.

The derivative of the average jump angle weighting, Eq. (15), can be taken with respect to $\beta$ to get

$$
\frac{\partial \bar{w}_{n}}{\partial \beta}=\int_{0}^{\pi} w_{n}(\Delta \theta) \frac{\partial P(\Delta \theta)}{\partial \beta} d \Delta \theta
$$

where

$$
\partial P(\Delta \theta) / \partial \beta=-\mathrm{h} \delta H\left(\tau^{*}\right) \delta\left[\Delta \theta-\Delta \tilde{\theta}(\mathbf{Q}] \mathrm{i}=-P_{H}(\Delta \theta),\right.
$$

is the derivative of the jump angle distribution. Here the energy fluctuation is evaluated at the time of each individual jump $\left(\tau^{*}\right)$. For a given $N V E$ trajectory, $\delta H\left(\tau^{*}\right)=$ $\delta H(0)$, but the same is not true for the energetic components, e.g., $\delta K E\left(\tau^{*}\right) 6=\delta K E(0)$.

\section{E. Frame Reorientation}

The frame reorientation time is determined by calculating the reorientational TCF for the $O_{d} \cdots O_{a}$ vector, which provides the local frame reorientation between successive jumps, 


$$
C_{\text {nframe }}(t)=\mathrm{h} P n[\sim e o o(0) \cdot \sim e o o(t)] \mathrm{i} \text { Hbond, }
$$

where the Hbond subscript on the average indicates that only molecules that have not switched $\mathrm{H}$-bond partners between times 0 and $t$ are included in the average. This leads to a time-dependent normalization for the TCF that is equal to the H-bond survival probability; this limits the times over which the TCF can be accurately calculated. The decay of $C_{n} f^{\text {frame }}(t)$ is well described by a biexponential function with the longer timescale equal to the frame time, $\tau_{n}$ frame.

The derivative of $C_{n}^{\text {frame }}(t)$ is completely analogous to that for $\mathrm{OH}$ reorientation given in Eq. (9) and can be fit through an expression of the form given in Eq. (10) with a sum over only two exponentials. In this approach, the frame activation energy,

$$
E_{a, n}^{\text {frame }}=-\frac{\partial \ln \left(1 / \tau_{n}^{\text {frame }}\right)}{\partial \beta}
$$

can then be extracted directly from the fitting of $C_{n}$ frame $(t)$ and its derivative TCF.

\section{METHODS}

The MD simulations were carried out using the Largescale Atomic/Molecular Massively Parallel Simulator (LAMMPS).$^{58}$ A fully-periodic simulation cell of side length $21.725311^{\circ} \mathrm{A}$ was filled with $343 \mathrm{SPC} / \mathrm{E}$ water molecules, ${ }^{59}$ corresponding to the $298.15 \mathrm{~K}$ experimental density of $0.997 \mathrm{~g} / \mathrm{cm}^{3}$. All simulations used a timestep of $1 \mathrm{fs}$, and the electrostatics from all simulations used the Particle-Particle-Particle Mesh Ewald summation method ${ }^{60,61}$ with a tolerance parameter of $1 \times 10^{-4}$. The SHAKE algorithm was used to hold the water bonds and angle rigid, the tolerance parameter was $1 \times 10^{-4}$. One $50 \mathrm{~ns}$ NVT trajectory was propagated with positions and momenta saved every 1 ps, yielding 50,000 configurations. The temperature of the simulation was $298.15 \mathrm{~K}$, with initial velocities selected from the Maxwell-Boltzmann distribution and the initial configuration generated by PACKMOL. ${ }^{62}$ The Nos'e-Hoover thermostat damping parameter was 100 fs. ${ }^{63,64}$

$$
\begin{aligned}
& \text { Order } \\
& \begin{array}{lllllll}
\hline n=1 & 3.141_{2} & 0.4512_{2} & 6.961_{4} & 16.91_{13} & 5.29_{3} & 4.93_{2}
\end{array} \\
& n=2 \begin{array}{lllllll}
3.141_{2} & 0.8819_{1} & 3.562_{2} & 5.97_{3} & 2.618_{7} & 2.231_{5}
\end{array}
\end{aligned}
$$

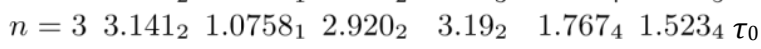

$$
\begin{aligned}
& W_{n} \tau_{n j u m p} \tau_{n \text { frame }} \quad \tau_{n} \quad \tau_{n E J M}
\end{aligned}
$$

TABLE I: Timescales (in ps) calculated from the jump dynamics, jump contribution, frame contribution, total reorientation, and EJM prediction are included along with the jump angle contribution, $w_{n}$; see the text for definitions. Subscripts represent the uncertainty in the final digit(s).

From each saved configuration, a 50 ps NVE simulation was run, from which the dynamical properties were evaluated. Configurations were saved every $10 \mathrm{fs}$, leading to 5000 total configurations per NVE trajectory; this is more than sufficient to resolve both the initial decay dynamics and the jump angle distribution. As has been noted in our prior works, each NVE trajectory has a different fluctuation in energy, $\delta H(0)$, from the average energy of the long trajectory. From each of these trajectories the $\mathrm{OH}$ reorientation, jump, and frame TCFs are calculated along with the jump angle distribution and each is also weighted by the energy fluctuations to obtain the contribution to their derivative with respect to $\beta$ as described in Sec. II. These individual correlation functions are then averaged across all the $N V E$ trajectories. All reported uncertainties correspond to a 95\% confidence interval according to the Student's $t$ distribution ${ }^{65}$ over an average of 10 blocks (each block representing 5,000 NVE trajectories).

\section{RESULTS AND DISCUSSION}

The timescales and activation energies for the $\mathrm{OH}$ reorientational dynamics were computed using the simulation approach described above along with those for the jump time, jump angle, and frame time components of the extended jump model. In the remainder of this paper, we present and discuss these results with the aim of both testing the ability of the EJM to accurately describe reorientation activation energies, and identifying the molecular origin of these activation energies.

\section{A. $\mathrm{OH}$ Reorientation}

We have calculated the $\mathrm{OH}$ reorientation TCF using Eq. (1) for $n=1-3$. The three time correlation functions are shown in Fig. 1 along with tri-exponential fits, Eq. (3). The $\tau_{n}$ timescales are provided in Table I. We note that the $n=2$ timescales (and activation energies) are in agreement with, but better converged than, our previous results.14,19,32,46

The activation energies of the reorientational timescales are obtained from the corresponding TCFs

Component $\quad E_{a, 0} \quad E_{a, n \Delta \theta} \quad E_{a, n j u m p} E_{a, n f r a m e}$




\begin{tabular}{ccccc}
\multicolumn{6}{c}{$n=1$} \\
\hline Total & $3.31_{5}$ & $0.302_{10}$ & $3.62_{5}$ & $3.62_{24}$ \\
KE & $0.96_{3}$ & $0.069_{7}$ & $1.03_{3}$ & $1.11_{13}$ \\
LJ & $-0.91_{4}$ & $-0.136_{10}$ & $-1.05_{4}$ & $-0.92_{16}$ \\
Coul & $3.27_{5}$ & $0.369_{14}$ & $3.64_{5}$ & $3.44_{28}$ \\
\hline \multicolumn{6}{c}{$n=2$} \\
\hline Total & $3.31_{5}$ & $0.129_{6}$ & $3.44_{5}$ & $3.66_{11}$ \\
KE & $0.96_{3}$ & $0.021_{4}$ & $0.98_{3}$ & $1.12_{8}$ \\
LJ & $-0.91_{4}$ & $-0.082_{5}$ & $-1.00_{4}$ & $-1.02_{15}$ \\
Coul & $3.27_{5}$ & $0.190_{8}$ & $3.46_{5}$ & $3.57_{24}$ \\
\hline \multicolumn{7}{c}{$n=3$} \\
\hline Total & $3.31_{5}$ & $0.003_{2}$ & $3.31_{5}$ & $3.68_{8}$ \\
KE & $0.96_{3}$ & $-0.012_{2}$ & $0.95_{3}$ & $1.09_{7}$ \\
LJ & $-0.91_{4}$ & $-0.032_{3}$ & $-0.95_{3}$ & $-1.05_{12}$ \\
Coul & $3.27_{5}$ & $0.047_{4}$ & $3.31_{5}$ & $3.65_{18}$
\end{tabular}

TABLE II: Activation energies and their kinetic energy

$(K E)$, Lennard-Jones ( $L J)$, and Coulombic ( $\mathrm{Cou}$ ) contributions (all in $\mathrm{kcal} / \mathrm{mol}$ ) for the components of the extended jump model for different order $n$ reorientational TCFs. Subscripts indicate the uncertainties in the final digit(s).

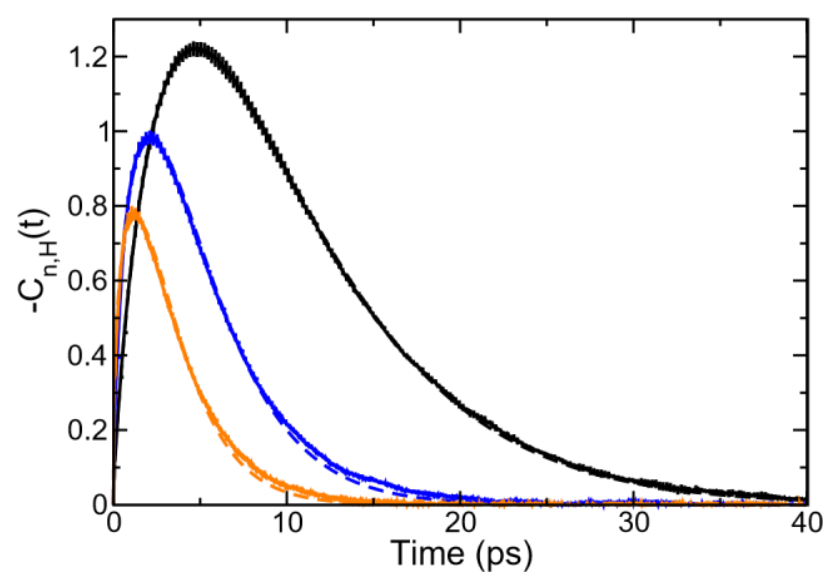

FIG. 3: Derivatives of the $\mathrm{OH}$ reorientational TCFs with respect to $\beta$, Eq. (9), for $\mathrm{H}_{2} \mathrm{O}$ at $298.15 \mathrm{~K}$ for

$n=1$ (black), 2 (blue), and 3 (orange); fits to Eq. (10) are shown as dashed lines of the same color.

weighted by the energy fluctuations, $\delta H(0)$, as given by Eq. (9). These derivative TCFs are shown in Fig. 3 for $n=$ $1-3$ along with their fits to the form in Eq. (10) based on a tri-exponential decay of $C_{n}(t)$. The fits provide an excellent description of the derivative TCFs and are used to obtain the $\mathrm{OH}$ reorientational activation energy for each order, $E_{a, n}$, from Eq. (6). The resulting values are provided in the two leftmost columns of

Table III.

We first use these results to examine the validity of the Debye model. In agreement with prior calculations, ${ }^{14,32,48,49}$ the $\tau_{n}$ reorientation time ratios deviate from the values predicted by the Debye rotational diffusion model: $\tau_{1} / \tau_{2}=2.02$ and $\tau_{1} / \tau_{3}=2.99$ are significantly smaller than the ratios of 3 and 6 , respectively, given by the diffusive model. The activation energies exhibit more modest differences and are not distinguishable outside the errors. This shows that the reorientation time ratios are expected to be approximately independent of temperature and thus that there is no temperature where these ratios would reach the ideal Debye diffusion values. While activation energies are not as instructive as a test of the Debye model compared to the reorientation timescales, they show that the deviation with respect to the Debye model is present over a broad temperature range in liquid water. In addition, a recent comparison of the activation energies of water reorientation, diffusion, and viscosity found deviations from the Debye-Stokes-Einstein description, which argues against this picture for water reorientation. ${ }^{23}$ Finally, the kinetic, Lennard-Jones, and Coulombic energy contributions to the reorientation activation energy, also reported in Table III, show that these different components are approximately independent of the order $n$. Their interpretation will be provided below.

We now proceed to test the ability of the extended jump model to describe the reorientation activation energy, and analyze its jump and frame components to provide a molecular understanding of reorientational activation energies.

\section{B. Jump Contribution to Reorientation}

As noted in Sec. IID, the jump contribution to the reorientational timescale has two components: the jump time and the distribution of jump angles. We consider each in turn.

1. H-bond Jump Times

The stable states TCF introduced in Sec. IID was used to calculate the H-bond jump time and its activation energy for water from the same simulations used to obtain the $\mathrm{OH}$ reorientational dynamics. The calculated $C_{a b}(t)$, Eq. (16), and its bi-exponential fit are shown in Fig. 4 a. The longer timescale is the jump time, $\tau_{0}$, and is found to be $3.142 \pm 0.002$ ps, which is in excellent agreement with previously reported results. ${ }^{18,32,46}$ Note that $\tau_{0}$ is the inverse of the rate constant $k_{0}$ for an $\mathrm{OH}$ to exchange one $\mathrm{H}$-bond acceptor for another and hence is independent of the order $n$ for the reorientational TCF.

The activation energy of the jump time is calculated from the $C_{a b, H}(t)$ correlation function given in Eq. (17), which is also plotted in Fig. 4a. By fitting this derivative TCF to a bi-exponential form analogous to Eq. (10), we 
obtain $\partial k_{0} / \partial \beta$ from which we find an activation energy of $E_{a, 0}=3.31 \pm 0.05 \mathrm{kcal} / \mathrm{mol}$. This is in good agreement with our previously reported values of $2.9 \mathrm{kcal} / \mathrm{mol}^{18,32}$ from an Arrhenius analysis and $3.2 \mathrm{kcal} / \mathrm{mol}$ from a direct
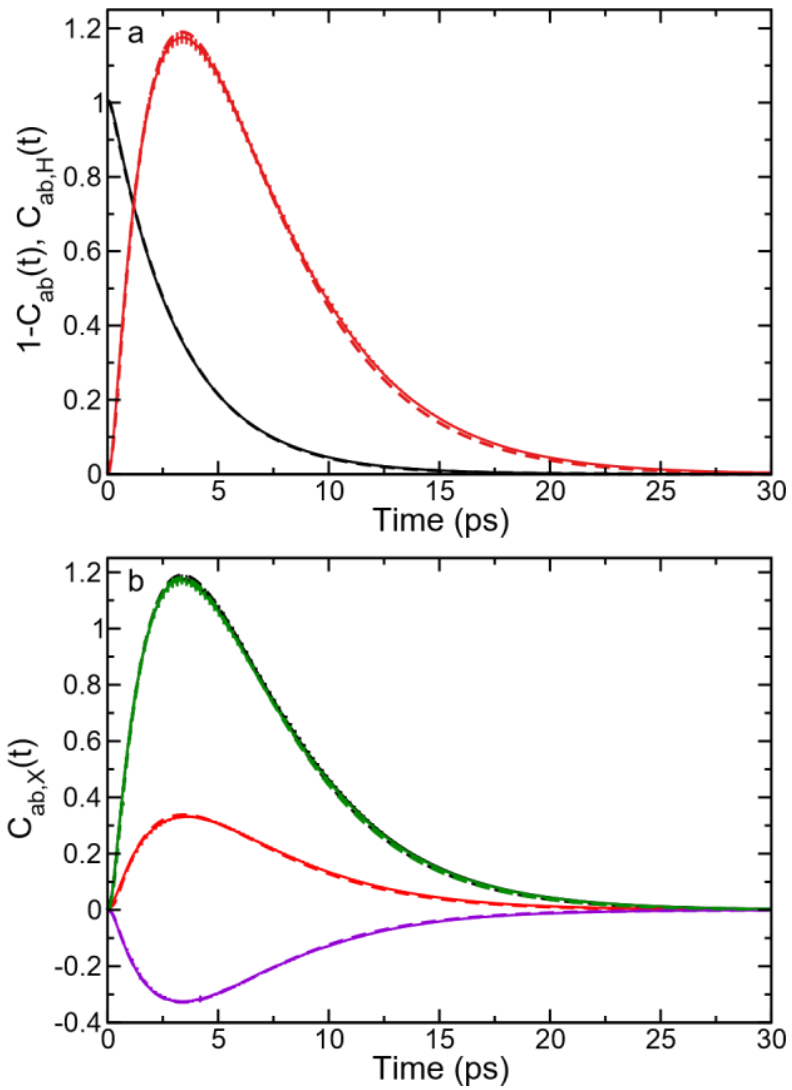

FIG. 4: a) Jump TCF, 1- $C_{a b}(t)$, (black) and its derivative, $C_{a b, H}(t)$, (red). b) The total energy (black), kinetic energy (red), Lennard-Jones (purple), and

Coulombic (green) contributions to the derivative TCF.

Calculated TCFs are shown as solid lines and fits as dashed lines of the same color.

fluctuation theory calculation. ${ }^{18}$ We note that the nonArrhenius temperature dependence of water dynamics around ambient conditions makes the results of Arrhenius analyses sensitive to the chosen temperature range. ${ }^{21}$ This jump time activation energy is lower than that for the $\mathrm{OH}$ reorientation timescale (independent of the order). This indicates that the latter, which is more easily measured, cannot be used alone to determine the former.

As discussed in Sec. IIA, the fluctuation theory approach provides a rigorous decomposition of the activation energy. We applied this approach to the jump time TCF using the energy components given in Eq. (11) and the results are shown in Fig. $4 \mathrm{~b}$. These are also fit to a bi-exponential form of Eq. (10) to extract activation energies associated with each contribution to $E_{a, 0}$ as in Eq. (12). The activation energies extracted in this way are reported in Table II.

The results in Fig. 4b and Table II are consistent with our previous analyses of water diffusion coefficients,19,22,24 reorientational dynamics,19,21,22,24 and viscosity. ${ }^{23}$ Namely, the electrostatic contribution dominates with $E_{a}{ }^{{ } \text {oul }}{ }_{0}=3.27 \pm 0.05 \mathrm{kcal} / \mathrm{mol}$, nearly equal to the total activation energy. The kinetic energy contribution is also positive, but smaller $(0.96 \pm 0.03$ $\mathrm{kcal} / \mathrm{mol}$ ) and the Lennard-Jones potential energy contributes a negative activation energy on the same order as the kinetic contribution $(-0.91 \pm 0.004$ $\mathrm{kcal} / \mathrm{mol})$. However, this near cancellation of $E_{a, 0}^{K E}$ and $E_{a, 0}^{L J}$ is actually fortuitous and it can be seen that it is actually the Coulombic and Lennard-Jones contributions that are in competition. ${ }^{24}$

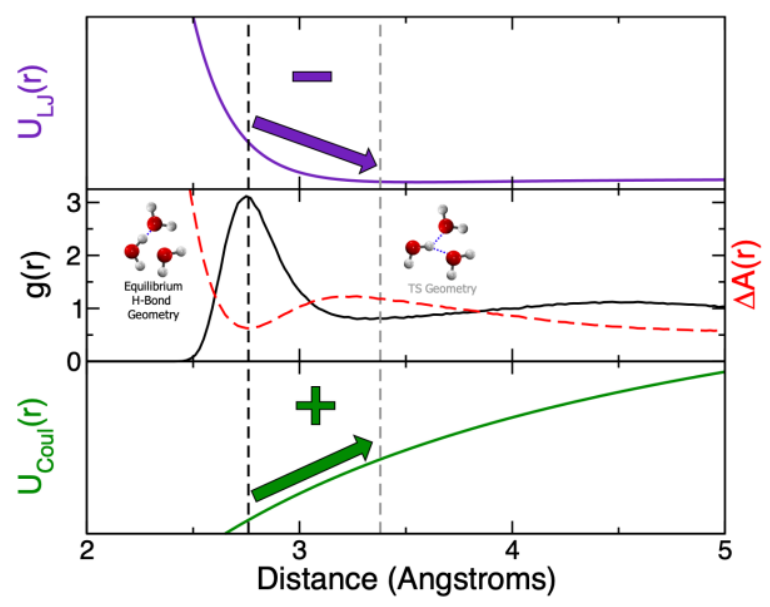

FIG. 5: Schematic illustration of the potential energy contributions to the jump activation energy. The "reactant" and transition state structures are shown (middle panel) in the context of the radial distribution function and free energy profile. The corresponding changes in the Lennard-Jones (top panel) and

Coulombic (bottom panel) interactions are depicted.

The physical explanation for this competition between the Coulombic and Lennard-Jones interactions can be understood by considering the chemical "reaction" involved in the H-bond jump. This is illustrated schematically in Fig. 5. In a typical H-bond the two water molecules sit high up on the repulsive wall of their mutual Lennard-Jones potential, held there by the attractive Coulombic interactions. To exchange $\mathrm{H}$-bond partners, the water molecules must increase their intermolecular distance, moving to the hydrogen bond exchange transition state (a bifurcated hydrogen-bond arrangement; as discussed in ref. 32 , it is unstable in ambient liquid water, in contrast to suggestions of stable 
overcoordinated water molecules in structures quenched from supercooled trajectories).66 This necessarily involves moving lower on the repulsive wall of the Lennard-Jones potential while simultaneous rising higher on the intermolecular electrostatic potential. This rise in the Coulombic energy and fall in the LennardJones potential associated with the process of an H-bond exchange leads to the positive and negative activation energy components, respectively. However, the electrostatic interactions are dominant and are thus quantitatively larger. The predominance of Coulombic interactions in the water jump and reorientation activation energies is consistent with the marked activation energy increase for water reorientation hydrogen-bonded to anions with increasing charge density, ${ }^{67}$ which has been explained by a transition-state $\mathrm{H}$-bond strength effect in the jump mechanism. ${ }^{34}$

\section{H-bond Jump Angle Distribution}

To fully calculate the activation energy for the jump reorientation component, it is also necessary to calculate the temperature-induced changes in the distribution of jump angles as described in Sec. IID.

We start by calculating the jump angle distribution. From each short $N V E$ trajectory, $P(\Delta \theta)$ was calculated using the first $\mathrm{H}$-bond exchange of every initially Hbonded $\mathrm{OH}$ group and then results were averaged across all of the trajectories. The distribution is shown in Fig. $6 \mathrm{a}$ and gives the average angular jump amplitude to be $\mathrm{h} \Delta \theta \mathrm{i}=70.31^{\circ} \pm 0.02^{\circ}$. This is in excellent agreement with the previously reported results by Laage and Hynes, ${ }^{32}$ which gave the average amplitude as $68^{\circ}$. With this jump angle distribution $w_{n}$ can be calculated using Eq. (15) for each order of the Legendre polynomial $n=1$, 2 , or 3; the resulting values are given in Table I.

The calculated jump angle distribution has two prominent features. The central feature peaks around $52.5^{\circ}$ and corresponds to H-bond exchanges in which the new H-bond acceptor is itself $\mathrm{H}$-bonded (either as a donor or acceptor) to the original H-bond acceptor for the $\mathrm{OH}$ of interest. ${ }^{68}$ The peak at larger jump angles is associated with new acceptors that have no $\mathrm{H}$-bond connection with the current acceptor.

A qualitative connection between the jump angle distribution and the water 00 radial distribution function can be suggested. At the jump transition state, a $\Delta \theta$ angle between $O^{a}, O^{d}$ and $O^{b}$ implies that the $O^{a} O^{b}$ distance is $200^{\ddagger} \sin (\Delta \theta / 2)$ where $O O^{\ddagger}$ is the $O^{d} O^{a, b}$ transition state distance. If one adopts the strong approximations that i) the $O^{a} O^{b}$ transition-state distribution is similar to the equilibrium 00 radial distribution function and ii) that the jump angle probability is only determined by the probability of the presence of the new acceptor $O^{b}$, thus ignoring the increase in jump barrier energy with $\Delta \theta$, the jump angle probability is approximately proportional to $g_{O O}\left(2 O O^{\ddagger} \sin (\Delta \theta / 2)\right) \sin (\Delta \theta)$.

This simplified geometric picture can explain the general shape of the jump angle distribution and suggests that the first peak in the jump angle distribution corresponds to the first peak in the 00 radial distribution function.

We have further calculated the derivative of the jump angle distribution with respect to $\beta,-P_{H}(\Delta \theta)$. This derivative distribution is obtained by the same process as that used to calculate $P(\Delta \theta)$, but the contribution of each jump angle is weighted by the energy fluctuation at the time of the jump, $\delta H\left(\tau^{*}\right)$. The derivative of the probability distribution is presented in Fig. 6b. To cal-
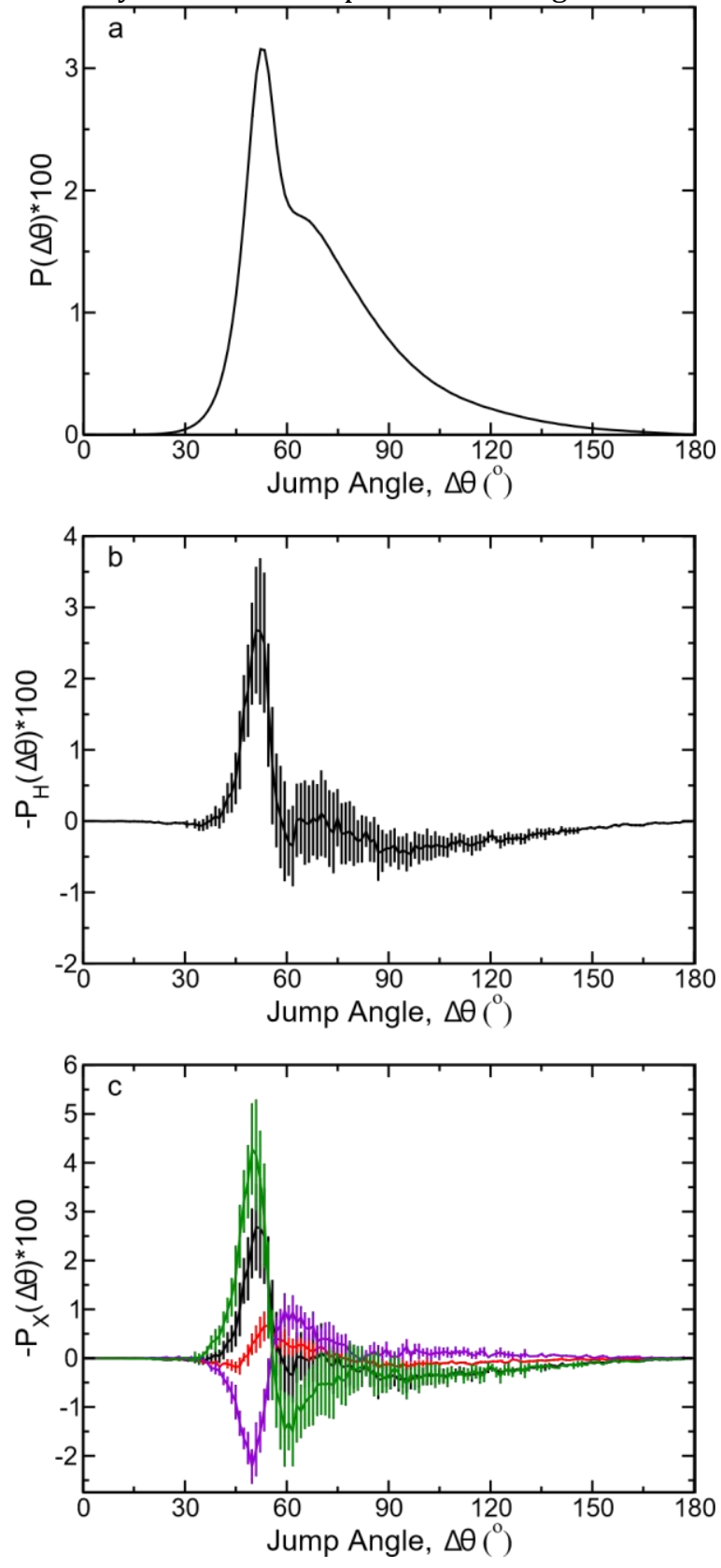
FIG. 6: a) The jump angle distribution, b) the derivative of the jump angle distribution, and c) the total (black), kinetic energy (red), Lennard-Jones

(purple) and Coulombic (green) contributions to the jump angle distribution derivative are presented.

culate the activation energy associated with changes in the jump angle distribution with temperature, $E_{a, n}^{\Delta \theta}$ in Eq. (19), we use Eqs. (15) and (20). These activation energies, given in Table II, are found to be $0.302 \pm 0.010$, $0.129 \pm 0.006$, and $0.003 \pm 0.002 \mathrm{kcal} / \mathrm{mol}$ for $n=1,2$, and 3 , respectively. Thus, they are at least an order of magnitude smaller than the jump time activation energy, $E_{a, 0}$, and represent only a small part of the overall jump contribution to the $\mathrm{OH}$ reorientational activation energy Ea,njump.

We have also calculated the decomposition of the jump angle distribution derivative with $\beta$, denoted as $-P_{X}(\Delta \theta)$ where $X=K E, L J$, or Coul. These derivative distributions are plotted in Fig. 6c and the contributions to the jump angle distribution activation energy are given in Table II. While the scale of the activation energy contributions decrease with increasing orders of the Legendre polynomial, the trends for the components are similar to that of the jump time. Namely, electrostatics represent the largest contribution, partially canceled by the smaller, negative Lennard-Jones component. Coulombic interactions disfavor large amplitude jumps when the temperature decreases, probably because in the transition state configurations for large-angle jumps the $\mathrm{OH}$ group that reorients loses its favorable electrostatic interactions with both the initial and final acceptors. The LJ potential disfavors small-angle jumps, presumably because they require small $O^{a} O^{b}$ distances that involve significant repulsive $\mathrm{LJ}$ interactions.

Interestingly, in contrast to the case of the radial distribution function and other static quantities that depend only on coordinates (not momenta), ${ }^{52}$ the kinetic energy contribution to jump angle distribution derivative is non-zero. This is a clear indication that the jump angle distribution is a dynamical quantity. Namely, $P(\Delta \theta)$ effectively depends on the coordinates before and after an H-bond exchange, which leads to a correlation between the system kinetic energy and the jump angle. The KE contribution is effectively zero for large-angle jumps but positive for small-angle ones, suggesting that these dynamical effects depend on the nature of the new acceptor.

Using the calculated activation energies of the jump angle distribution, $E_{a, n}^{\Delta \theta}$, and the characteristic jump timescale, $E_{a, 0}$, the total jump contribution to the $\mathrm{OH}$ reorientation activation energy, $E_{a, n}{ }^{\text {jump }}$, can be calculated from Eq. (19). These are found to be $3.62 \pm 0.05,3.44 \pm$
0.05 , and $3.31 \pm 0.05 \mathrm{kcal} / \mathrm{mol}$, respectively, for $n=1,2$, and 3. The full decomposition of these activation energies are provided in Table II. Note that the change in $E_{a, r^{j u m p}}$ with the order of the reorientational TCF is associated only with the jump angle distribution (which itself does not depend on $n$ ) reflecting different weighting of the large-amplitude angular jumps.

\section{Frame Contribution to Reorientation}

The final component of the EJM is the frame reorientation. We have calculated the frame reorientation TCF as described in Sec. IIE and the results are shown in

Fig. 7a. Because $C_{n \text {,frame }}(t)$ can only be obtained from $\mathrm{OH}$ groups that have not exchanged their H-bond acceptor, the TCF can only be calculated for limited times (up to delays on the order of the H-bond jump time). In practice, we find that the time range over which data can
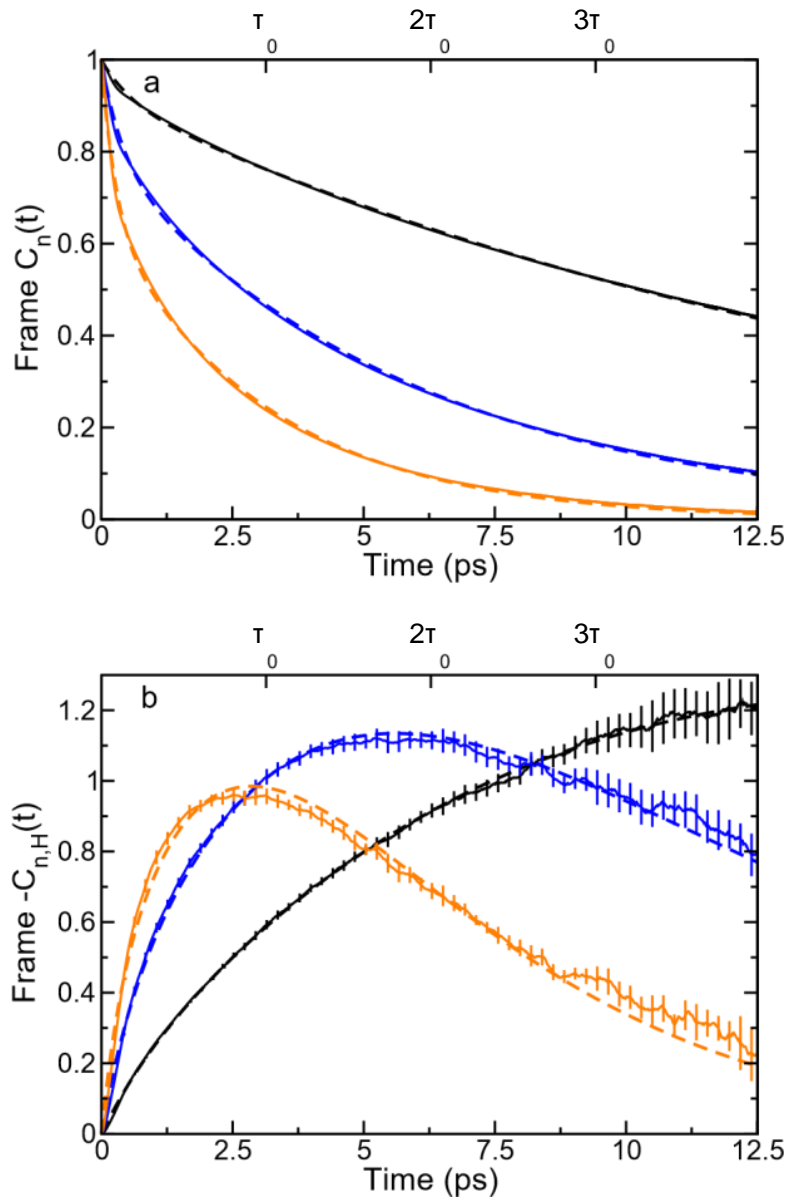

FIG. 7: a) Frame reorientational TCF, $C_{n \text {,frame }}(t)$ for $n=1$ (black), 2 (blue), and 3 (orange), and b)

Corresponding derivative TCFs, $C_{n, \text { frame, } H}(t)$. Fits are shown as dashed lines in the same color. 
be reliably obtained is approximately four times the characteristic jump time $\tau_{0}$, or $12.5 \mathrm{ps}$. At this time, fewer than $2 \%$ of the originally $\mathrm{H}$-bonded $\mathrm{OH}$ moieties have not switched partners and are thus still contributing to

$C_{n, \text { frame }}(t)$.

The calculated $C_{n, \text { frame }}(t)$ are well described by a biexponential fit, also shown in Fig. 7a, in which the longer time is the frame reorientation time. We find $\tau_{n}{ }^{\text {frame }}=16.91 \pm 0.13,5.97 \pm 0.03$, and $3.19 \pm 0.02$ ps for $n=$ 1,2 , and 3, respectively. ${ }^{69}$ The $n=1$ and 2 values are in good agreement with the $\tau_{1}^{\text {frame }}=15.5 \mathrm{ps}$ and $\tau_{2}^{\text {frame }}=5$. 6 ps previously obtained by Laage and Hynes. ${ }^{32}$

The derivatives of the frame reorientation TCFs,

$$
\begin{aligned}
\frac{\partial C_{n}^{\text {frame }}(t)}{\partial \beta} & =-\left\langle\delta H(0) P_{n}\left[\vec{e}_{O O}(0) \cdot \vec{e}_{O O}(t)\right]\right\rangle_{\text {Hbond }} \\
& \equiv C_{n, \text { frame }, H}(t),
\end{aligned}
$$

were also calculated and are presented in Fig. $7 \mathrm{~b}$. We find that the frame activation energies are $3.62 \pm 0.24$, $3.66 \pm 0.11$, and $3.68 \pm 0.08 \mathrm{kcal} / \mathrm{mol}$ for $n=1,2$, and 3 , respectively.

It has been suggested that, while the mechanism for $\mathrm{OH}$ reorientation is not diffusive, the frame reorientation is much closer to the ideal diffusive behavior. ${ }^{32}$ This rotation likely involves, as elementary steps, Hbond exchanges between the central H-bonded partners and the surrounding waters in their first solvation shell (while maintaining the central H-bond). We can examine whether collectively these jumps lead to Debye rotational diffusion for the frame reorientation using the two essential predictions of the timescales and activation energies made by the Debye model in Eq. (2). Namely, in terms of the frame reorientation timescales calculated here, we find

$$
\tau_{1}^{\text {frame }} / \tau_{2}^{\text {frame }}=2.83, \tau_{1}^{\text {frame }} / \tau_{3}^{\text {frame }}=5.30 \text {, }
$$

and

$\tau_{2}^{\text {frame }} / \tau_{3}^{\text {frame }}=1.87$, which are in reasonable accord with the Debye predictions of 3,6 , and 2, respectively. These timescale ratios thus suggest that the contributions from multiple $\mathrm{H}$-bond exchanges with the solvating water molecules leads to primarily diffusive rotational dynamics for the frame.

The activation energies obtained for frame reorientation, Table II, are the same within statistical error, which is also consistent with the Debye model. However, the example of $\mathrm{OH}$ reorientation discussed in Sec. IVA, suggests the activation energies may not be instructive for testing the rotational diffusion model.

The derivative TCF for frame reorientation, $C_{n, \text { frame, } H}(t)$ can also be decomposed in terms of contributions from the kinetic, Lennard-Jones, and Coulombic energies. These results are shown in Fig. 8 and the derived activation energies are given in Table II. The magnitudes and signs of the different components of the frame activation energy follow the same trends observed for the jump time. In addition, as is true for the total $E_{a, n}$ frame, each contribution is nearly independent of the order $n$ of the reorientational TCF.

\section{Tests of the Extended Jump Model}

In this Section, we address two important features of the extended jump model: 1) its ability to quantitatively predict $\mathrm{OH}$ reorientation activation energies, and their components associated with different interactions and motions, and 2) the relative contributions of the jump and frame contributions to temperature dependence of the $\mathrm{OH}$ reorientation time. The latter will provide a novel insight in the molecular origins of the reorientation activation energy.

\section{Prediction of $\mathrm{OH}$ Reorientation Times}

The extended-jump model has been demonstrated to describe the timescales and mechanisms of water reorientation in neat water ${ }^{14,32}$ and the jumps have been characterized via two-dimensional infrared spectroscopy experiments in aqueous salt solutions. ${ }^{70,71}$ In particular, Laage and Hynes showed that the overall reorientation
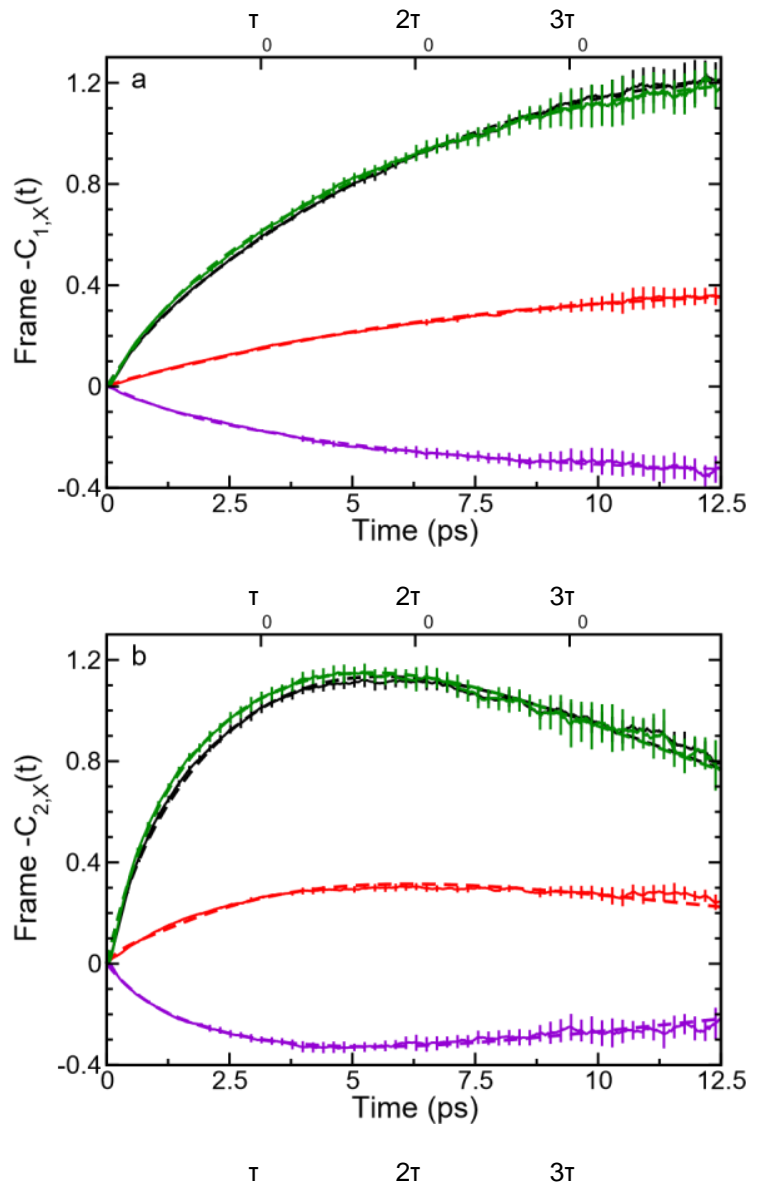


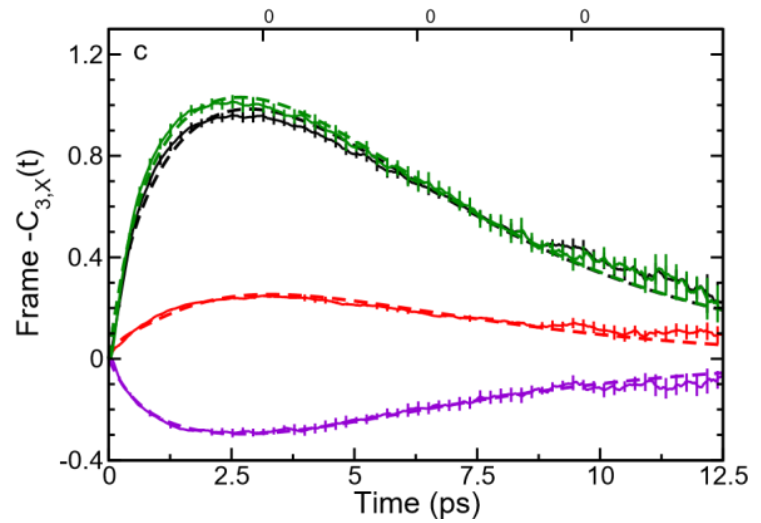

FIG. 8: Decomposition of the derivative frame reorientation TCF, $C_{n, \text { frame } H}(t)$, for a) $n=1, \mathrm{~b}$ ) $n=2$, and c) $n=3$. Contributions from kinetic (red), Lennard-Jones (purple), and Coulombic (green) energies are compared to the total (black). Fits are shown as dashed lines of the same color.

rate constant is the sum of the jump and frame reorientation rate constants, Eq. (4). In Table I we have included these contributions, $\tau_{n}{ }^{\text {jump }}$ and $\tau_{n}^{\text {frame }}$, as well as the predicted $\mathrm{OH}$ reorientation timescale, $\tau_{n}^{E J M}$. It can be seen that the EJM reasonably predicts the $\mathrm{OH}$ reorientational timescales for all three orders of the TCF and the results are in good agreement with those jump axis distribution and of a decoupling between the jumps of the two $\mathrm{OH}$ groups in the same water molecule) or by some more fundamental issue with the jump mechanism.

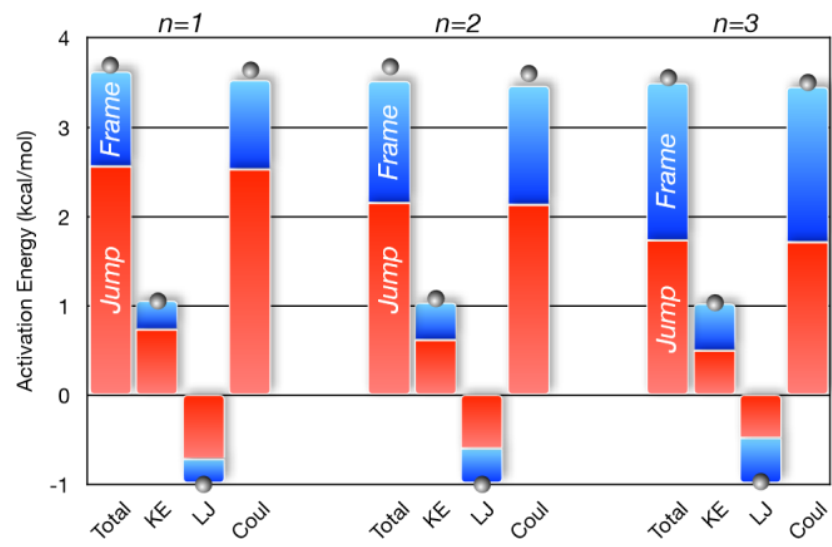

FIG. 9: Contributions to the $\mathrm{OH}$ reorientation activation energy for $n=1,2$, and 3 (gray spheres) are compared to the EJM predictions (bars) divided into the jump (red bars) and frame (blue bars) weighted components in Eq. (7).

\section{Prediction of $\mathrm{OH}$ Reorientation Activation Energies}

\begin{tabular}{|c|c|c|c|c|c|c|c|}
\hline \multirow[t]{2}{*}{ Component } & \multirow{2}{*}{$\begin{array}{c}E_{a, n} \\
\text { Calculated }\end{array}$} & & \multicolumn{4}{|c|}{$=$ Weighted Jump + Weighted Frame $=$} & \multirow{2}{*}{$\begin{array}{c}E_{a, n E J M} \\
\text { Predicted }\end{array}$} \\
\hline & & & Contribution & & Contribution & & \\
\hline \multicolumn{8}{|l|}{$n=1$} \\
\hline Total & $3.70_{11}$ & $=$ & $2.56_{4}$ & + & $1.06_{7}$ & $=$ & $3.62_{8}$ \\
\hline$K E$ & 1.069 & $=$ & $0.73_{2}$ & + & $0.32_{4}$ & $=$ & $1.05_{4}$ \\
\hline$L J$ & $-1.01_{9}$ & $=$ & $-0.74_{3}$ & + & $-0.27_{4}$ & $=$ & $-1.01_{6}$ \\
\hline Coul & $3.65_{8}$ & $=$ & $2.58_{4}$ & + & 1.009 & $=$ & 3.589 \\
\hline \multicolumn{8}{|l|}{$n=2$} \\
\hline Total & $3.69_{7}$ & $=$ & $2.15_{3}$ & + & $1.37_{5}$ & $=$ & $3.52_{5}$ \\
\hline$K E$ & $1.08_{5}$ & $=$ & $0.62_{2}$ & + & $0.42_{3}$ & $=$ & $1.03_{4}$ \\
\hline$L J$ & $-1.01_{7}$ & $=$ & $-0.63_{1}$ & + & $-0.38_{5}$ & $=$ & $-1.00_{6}$ \\
\hline Coul & $3.61_{10}$ & $=$ & $2.17_{3}$ & + & $1.33_{9}$ & $=$ & $3.50_{10}$ \\
\hline \multicolumn{8}{|l|}{$n=3$} \\
\hline Total & $3.56_{7}$ & $=$ & $1.73_{3}$ & + & $1.76_{4}$ & $=$ & $3.49_{5}$ \\
\hline$K E$ & $1.03_{5}$ & $=$ & $0.50_{2}$ & + & $0.52_{3}$ & $=$ & $1.01_{4}$ \\
\hline$L J$ & $-0.98_{7}$ & $=$ & $-0.49_{2}$ & + & $-0.50_{5}$ & $=$ & $-1.00_{6}$ \\
\hline Coul & $3.51_{11}$ & $=$ & $1.73_{3}$ & + & $1.74_{9}$ & $=$ & $3.47_{9}$ \\
\hline
\end{tabular}

TABLE III: The additivity of the activation energies (all in $\mathrm{kcal} / \mathrm{mol}$ ) within the EJM is presented. The directly calculated $\mathrm{OH}$ reorientation compared for each $n$ to the

$$
E_{a, n}^{E J M}
$$
activation energy $\left(E_{a, n}\right)$ and its different energetic contributions are EJM prediction () and its weighted jump and frame reorientation components in Eq. (7).

previously reported. ${ }^{14,32}$ We note, however, that the EJM prediction is not exact, and the simple consideration of the reorientation times cannot decide whether the small residual discrepancy is caused by some of the EJM approximations (e.g., the assumptions of an isotropic
Here, we have presented a different approach for examining the extended jump model by examining its predictions for the temperature dependence of the $\mathrm{OH}$ reorientational timescales. Specifically, we have used our calculated results for the activation energies of the jump 
angle distribution, the jump time, and the frame reorientation time to calculate the $\mathrm{OH}$ reorientation activation energy predicted by the EJM in Eq. (7). We now compare these predictions to the directly computed $\mathrm{OH}$ reorientation activation energies. The fluctuation theory approach avoids potential numerical inaccuracies associated with choosing a range of temperatures for an Arrhenius analysis as well as any effects of intrinsic nonArrhenius behavior. This issue is particularly critical in liquid water, where these non-Arrhenius effects are especially pronounced for dynamical properties. ${ }^{8,15-17}$

In Table III and Fig. 9, we compare the calculated activation energy for each reorientational timescale $\tau_{n}$ to the EJM prediction, which is further broken down into the contributions from $\mathrm{H}$-bond jumps and frame reorientation. Note that the jump and frame contributions to the activation energies include the reweighting by the ratio of the timescales as seen in Eq. (7). The results clearly show that the EJM accurately predicts the activation energies of water $\mathrm{OH}$ reorientation for all three orders of the TCF. As activation energies are a commonly used measure of the temperature dependence of timescales, this indicates that the EJM not only accurately predicts the $\mathrm{OH}$ reorientation time itself, but also the temperature dependence of the reorientation time. This is true for all orders of the reorientational TCF, but most importantly, it is accurate for the $n=2$ order which is experimentally accessible via both NMR and pump-probe infrared anisotropy experiments.

3. Jump and Frame Contributions to $E_{a, n}$

It is interesting to examine the relative contributions of the jump and frame mechanisms to the $\mathrm{OH}$ reorientation activation energy. The jump and frame contributions to the $n^{\text {th }}$-order reorientational time activation energies are given in Table II. As noted above, the frame reorientation activation energy is $E_{a, n}$ frame' 3.65 $\mathrm{kcal} / \mathrm{mol}$ independent of the order. This is similar in magnitude to the jump contribution, which includes the temperature dependence of both the jump time and the jump angle distribution. For $n=1$, the overall jump activation energy, Eq. (19), is effectively the same as that for frame reorientation $\left(E_{a, n^{j u m p}}=3.62 \pm 0.05 \mathrm{kcal} / \mathrm{mol}\right)$. How these two combine to predict the $\mathrm{OH}$ reorientation activation energy depends on the relative jump and frame timescales as given in Eq. (7). Because the jump contribution to the reorientation time is faster than the frame time, $\tau_{n}{ }^{\text {jump }}<\tau_{n}{ }^{\text {frame }}$ (see Table I), the $\mathrm{OH}$ reorientation time has a larger contribution from the jumps. This is illustrated in Table III and Fig. 9 where the weighted jump and frame contributions to $\tau_{n}$ given in Eq. (7) are shown. For the $n=1$ reorientational TCF, the weighted jump contribution is $\sim 2.5$ times larger than the weighted frame contribution.

As the order increases, the jump and frame contributions to $\tau_{n}$ both decrease but also get closer, as shown in Table I. At the same time, the frame activation energy is independent of $n$ while the jump contribution activation energy decreases modestly with $n$; see Table II. The effect of these two trends is that the weighted frame contribution to the $\mathrm{OH}$ reorientation activation energy grows as the weighted jump contribution shrinks. As given in Table III and shown in Fig. 9, for $n=2$, the weighted jump contribution is only 1.5 times the weighted frame contribution and, for $n=3$, the two are equal. Thus, the relative importance of frame reorientation and $\mathrm{H}$-bond jumps to the $\mathrm{OH}$ reorientational activation energy $E_{a, n}$ depends on the order $n$. And it does so primarily because of the difference in how $\tau_{n}{ }^{\text {frame }}$ decreases more quickly with $n$ than does $\tau_{n}{ }^{\text {jump }}$.

The close quantitative similarity of the activation energies for the jump and frame contributions to $\mathrm{OH}$ reorientation, $E_{a, n}{ }^{\text {jump }}$ and $E_{a, n}$ frame, indicates that their relative importance in determining $E_{a, n}$ will not be strongly temperature dependent. In other words, the effect of H-bond jumps should be the dominant contribution to both $\tau_{n}$ and $E_{a, n}$ for $n=1$ or 2 over a significant range of temperatures (at least outside the deeply supercooled regime where non-Arrhenius effects are important.)

While it is important that the EJM description of water reorientation accurately predicts the activation energy, it is even more critical that it does so for the right reasons. We have tested this using the decomposition of the calculated $E_{a, n}$ into kinetic, Lennard-Jones, and Coulombic energy contributions in direct comparison to the EJM predictions. These results are also included in Table III and Fig. 9. As with the total activation energy, each component also correctly predicts the reorientation activation energy contribution from that component. Thus, the EJM not only accurately describes the activation energy but also the physical interpretation of its origins.

\section{CONCLUSION}

This work demonstrates an application of the fluctuation theory for dynamics to the extended jump model of water reorientation. We have calculated the activation energies of the jump reorientation time, the distribution of jump angles, and the frame reorientation time and used them to predict the $\mathrm{OH}$ reorientation time 
in liquid water for multiple orders of the reorientation correlation function. Importantly, the activation energies extracted from both the actual and predicted $\mathrm{OH}$ reorientation agree, but they are also in good agreement with experimental results from pump-probe infrared anisotropy experiments, ${ }^{3-5}$ supporting the EJM as the physical model underlying water reorientation. Furthermore, the activation energy decomposition indicates that the EJM correctly determines the activation energies for the right reasons as the predicted and the actual decomposition of the $\mathrm{OH}$ reorientation activation energy are also in excellent agreement.

The frame reorientation times and activation energies were examined probe the nature of this reorientation. The ratio of timescales of different orders of the reorientational TCF strongly indicate that the frame reorientation is reasonably described by Debye rotational diffusion. This rotational diffusion is likely caused by Brownian orientational motions induced in the frame of the unbroken hydrogen bonds by exchanges within the first solvation shells of the H-bond partners. It was found that not only the total activation energy, but also the decomposition of the activation energy, was unchanging with the order of the Legendre polynomial; while this result is predicted by the Debye model of rotational diffusion, this behavior of the activation energies does not appear to be significantly different from the predictions of the EJM.

In all, the work presented here provides strong evidence for the EJM description of $\mathrm{OH}$ reorientation. Furthermore, for the first time, the contributions to water reorientation from each component of the activation energy has been explained in terms of jump and frame component activation energies. The methods presented here are general and could be easily extended to reorientation of water molecules around a solute or in the first hydration layer of a biomolecule.

\section{ACKNOWLEDGMENTS}

The authors would like to thank Professor Brian B. Laird for many useful discussions. This work was supported by the National Science Foundation under Grant No. CHE-1800559. This material is based on the work supported by the National Science Foundation Graduate Research Fellowship under Grant Nos. 1540502 and 1451148 as well as the National Science Graduate Research Opportunities Worldwide Program. The calculations were performed at the University of Kansas Center for Research Computing (CRC).

DATA AVAILABILITY
The data that support the findings of this study are available from the corresponding author upon reasonable request.

${ }^{1}$ E. Lang and H.-D. Lu"demann, "Pressure and temperature dependence of the longitudinal proton relaxation times in supercooled water to $87^{\circ} \mathrm{C}$ and 2500 bar," J. Chem. Phys. 67, 718-723 (1977).

2J. Qvist, C. Mattea, E. P. Sunde, and B. Halle, "Rotational dynamics in supercooled water from nuclear spin relaxation and molecular simulations," J. Chem. Phys. 136, 204505 (2012).

${ }^{3}$ C. Petersen, K. J. Tielrooij, and H. J. Bakker, "Strong temperature dependence of water reorientation in hydrophobic hydration shells," J. Chem. Phys. 130, 214511 (2009).

${ }^{4}$ R. A. Nicodemus, K. Ramasesha, S. T. Roberts, and A. Tokmakoff, "Hydrogen bond rearrangements in water probed with temperaturedependent 2D IR," J. Phys. Chem. Lett. 1, 1068- 1072 (2010).

${ }^{5}$ R. A. Nicodemus, S. A. Corcelli, J. L. Skinner, and A. Tokmakoff, "Collective hydrogen bond reorganization in water studied with temperature-dependent ultrafast infrared spectroscopy," J. Phys. Chem. B 115, 5604-5616 (2011).

${ }^{6} \mathrm{~F}$. Perakis and P. Hamm, "Two-Dimensional Infrared Spectroscopy of Supercooled Water," J. Phys. Chem. B 115, 5289- 5293 (2011).

${ }^{7}$ C. Rønne, L. Thrane, P.-O. Astrand, A. Wallqvist, K. V. Mikkelsen, and S. R. Keiding, "Investigation of the temperature dependence of dielectric relaxation in liquid water by $\mathrm{THz}$ reflection spectroscopy and molecular dynamics simulation," J. Chem. Phys. 107, 5319-5331 (1997).

${ }^{8}$ C. H. Cho, J. Urquidi, S. Singh, and G. W. Robinson, "Thermal Offset Viscosities of Liquid $\mathrm{H}_{2} \mathrm{O}, \mathrm{D}_{2} \mathrm{O}$, and $\mathrm{T}_{2} \mathrm{O}$," J. Phys. Chem. B 103, 19911994 (1999).

${ }^{9}$ L. D. Eicher and B. J. Zwolinski, "High-precision viscosity of supercooled water and analysis of the extended range temperature coefficient," J. Phys. Chem. 75, 2016-2024 (1971).

${ }^{10} \mathrm{~L}$. A. Woolf, "Tracer Diffusion of Tritiated Water (THO) in Ordinary Water $\left(\mathrm{H}_{2} \mathrm{O}\right)$ under Pressure," J. Chem. Soc., Faraday Trans. 1 71, 784-796 (1975).

${ }^{11} \mathrm{~K}$. Krynicki, C. D. Green, and D. W. Sawyer, "Pressure and Temperature Dependence of Self-diffusion in Water," Faraday Discuss. Chem. Soc. 66, 199-208 (1978).

${ }^{12}$ J. Teixeira, M.-C. Bellissent-Funel, S. H. Chen, and A. J. Dianoux, "Experimental determination of the nature of diffusive motions of water molecules at low temperatures," Phys. Rev. A 31, 1913-1917 (1985).

${ }^{13}$ J. Qvist, H. Schober, and B. Halle, "Structural dynamics of supercooled water from quasielastic neutron scattering and molecular simulations." J. Chem. Phys. 134, 144508 (2011).

${ }^{14} \mathrm{D}$. Laage and J. T. Hynes, "A molecular jump mechanism of water reorientation," Science 311, 832-835 (2006).

${ }^{15}$ P. Gallo, K. Amann-Winkel, C. A. Angell, M. A. Anisimov, F. Caupin, C. Chakravarty, E. Lascaris, T. Loerting, A. Z. Panagiotopoulos, J. Russo, J. A. Sellberg, H. E. Stanley, H. Tanaka,

C. Vega, L. Xu, and L. G. M. Pettersson, "Water: A Tale of Two Liquids," Chem. Rev. 116, 7463-7500 (2016).

${ }^{16}$ Y. Xu, N. G. Petrik, R. S. Smith, B. D. Kay, and G. A. Kimmel, "Growth rate of crystalline ice and the diffusivity of supercooled water from 126 to 262 K." Proc. Natl. Acad. Sci. 113, 14921- 14925 (2016).

${ }^{17}$ N. J. Hestand and J. L. Skinner, "Perspective: Crossing the Widom line in no man's land: Experiments, simulations, and the location of the liquid-liquid critical point in supercooled water," J. Chem. Phys. 149, 140901 (2018).

${ }^{18} \mathrm{O}$. O. Mesele and W. H. Thompson, "Removing the barrier to the calculation of activation energies," J. Chem. Phys. 145, 134107 (2016). 
${ }^{19}$ Z. A. Piskulich, O. O. Mesele, and W. H. Thompson, "Removing the barrier to the calculation of activation energies: Diffusion coefficients and reorientation times in liquid water," J. Chem. Phys. 147, 134103 (2017).

${ }^{20} \mathrm{Z}$. A. Piskulich, O. O. Mesele, and W. H. Thompson, "Expanding the calculation of activation volumes: Self-diffusion in liquid water," J. Chem. Phys. 148, 134105 (2018).

${ }^{21 Z}$. A. Piskulich and W. H. Thompson, "The activation energy for water reorientation differs between IR pump-probe and NMR measurements," J. Chem. Phys. 149, 164504 (2018).

22Z. A. Piskulich, O. O. Mesele, and W. H. Thompson, "Activation energies and beyond," J. Phys. Chem. A 123, 7185-7194 (2019). ${ }^{23}$ C. Mendis, Z. A. Piskulich, and W. H. Thompson, "Tests of the Stokes-Einstein relation through the shear viscosity activation energy of water," J. Phys. Chem. B. 123, 5857-5865 (2019).

${ }^{24} \mathrm{Z}$. A. Piskulich and W. H. Thompson, "The dynamics of supercooled water can be predicted from room temperature simulations," J. Chem. Phys. 152, 074505 (2020).

${ }^{25} \mathrm{G}$. Stirnemann and D. Laage, "Communication: On the origin of the non-Arrhenius behavior in water reorientation dynamics," J Chem Phys 137, 031101 (2012).

${ }^{26} \mathrm{H}$. J. Bakker and J. L. Skinner, "Vibrational spectroscopy as a probe of structure and dynamics in liquid water," Chem. Rev. 110, 1498-1517 (2009).

${ }^{27}$ Y. S. Lin, P. A. Pieniazek, M. Yang, and J. L. Skinner, "On the calculation of rotational anisotropy decay, as measured by ultrafast polarizationresolved vibrational pump-probe experiments," J. Chem. Phys. 132, 174505 (2010).

${ }^{28}$ C. J. Fecko, J. J. Loparo, S. T. Roberts, and A. Tokmakoff, "Local hydrogen bonding dynamics and collective reorganization in water: ultrafast infrared spectroscopy of $\mathrm{HOD} / \mathrm{D}_{2} \mathrm{O}$," J. Chem. Phys. 122, 54506 (2005).

${ }^{29}$ H. J. Bakker, Y. L. A. Rezus, and R. L. A. Timmer, "Molecular reorientation of liquid water studied with femtosecond midinfrared spectroscopy," J. Phys. Chem. A 112, 11523-11534 (2008). 30D. E. Moilanen, E. E. Fenn, Y.-S. Lin, J. L. Skinner, B. Bagchi, and M. D. Fayer, "Water inertial reorientation: Hydrogen bond strength and the angular potential," Proc. Natl. Acad. Sci. 105, 5295-5300 (2008).

${ }^{31}$ E. N. Ivanov, "Theory of rotational brownian motion," Sov. Phys. JETPUSSR 18, 1041-1045 (1964).

${ }^{32} \mathrm{D}$. Laage and J. T. Hynes, "On the molecular mechanism of water reorientation." J. Phys. Chem. B 112, 14230-14242 (2008).

${ }^{33}$ D. Laage, G. Stirnemann, F. Sterpone, R. Rey, and J. T. Hynes, "Reorientation and allied dynamics in water and aqueous solutions," Annu. Rev. Phys. Chem. 62, 395-416 (2011).

${ }^{34}$ J. Boisson, G. Stirnemann, D. Laage, and J. T. Hynes, "Water reorientation dynamics in the first hydration shells of $\mathrm{F}-$ and I-," Phys. Chem. Chem. Phys. 13, 19895 (2011).

${ }^{35} \mathrm{G}$. Stirnemann, E. Wernersson, P. Jungwirth, and D. Laage, "Mechanisms of acceleration and retardation of water dynamics by ions," J. Am. Chem. Soc. 135, 11824-11831 (2013).

${ }^{36} \mathrm{D}$. Laage and G. Stirnemann, "Effect of ions on water dynamics in dilute and concentrated aqueous salt solutions." J. Phys. Chem. B 123, 3312-3324 (2019).

${ }^{37}$ D. Laage, G. Stirnemann, and J. T. Hynes, "Why water reorientation slows without iceberg formation around hydrophobic solutes," J. Phys. Chem. B 113, 2428-2435 (2009).

${ }^{38}$ F. Sterpone, G. Stirnemann, J. T. Hynes, and D. Laage, "Water hydrogen-bond dynamics around amino acids: The key role of hydrophilic hydrogen-bond acceptor groups," J. Phys. Chem. B 114, 2083-2089 (2010).

${ }^{39}$ G. Stirnemann, S. R.-V. Castrill'on, J. T. Hynes, P. J. Rossky, P. G. Debenedetti, and D. Laage, "Non-monotonic dependence of water reorientation dynamics on surface hydrophilicity: Competing effects of the hydration structure and hydrogen-bond strength," Phys. Chem. Chem. Phys. 13, 19911-19917 (2011).

${ }^{40}$ S. Xiao, F. Figge, G. Stirnemann, D. Laage, and J. A. McGuire, "Orientational dynamics of water at an extended hydrophobic interface," J. Am. Chem. Soc. 138, 5551-5560 (2016).

${ }^{41} \mathrm{D}$. Laage, T. Elsaesser, and J. T. Hynes, "Water dynamics in the hydration shells of biomolecules," Chem. Rev. 117, 10694-10725 (2017).

${ }^{42}$ A. C. Fogarty, F.-X. Coudert, A. Boutin, and D. Laage, "Reorientational dynamics of water confined in zeolites," ChemPhysChem 15, 521529 (2014).

${ }^{43}$ S. Abel, N. Galamba, E. Karakas, M. Marchi, W. H. Thompson, and D. Laage, "On the structural and dynamical properties of DOPC reverse micelles," Langmuir 32, 10610-10620 (2016).

${ }^{44} \mathrm{D}$. Laage and W. H. Thompson, "Reorientation dynamics of nanoconfined water: Power-law decay, hydrogen-bond jumps, and test of a two-state model," J. Chem. Phys. 136, 044513

(2012).

${ }^{45}$ A. C. Fogarty, E. Dubou'e-Dijon, D. Laage, and W. H. Thompson, "Origins of the non-exponential reorientation dynamics of nanoconfined water," J. Chem. Phys. 141, 18C523 (2014).

${ }^{46}$ A. A. Vartia, K. R. Mitchell-Koch, G. Stirnemann, D. Laage, and W. H. Thompson, "On the reorientation and hydrogen-bond dynamics of alcohols," J. Phys. Chem. B 115, 12173-12178 (2011). ${ }^{47}$ O. O. Mesele, A. A. Vartia, D. Laage, and W. H. Thompson,

"Reorientation of isomeric butanols: The multiple effects of steric bulk arrangement on hydrogen-bond dynamics," J. Phys. Chem. B 120, 1546-1559 (2016).

${ }^{48} \mathrm{~A}$. Rahman and F. Stillinger, "Molecular dynamics study of liquid water," J. Chem. Phys. 55, 3336-3359 (1971).

${ }^{49} \mathrm{P}$. J. Rossky and M. Karplus, "Solvation. a molecular dynamics study of a dipeptide in water," J. Am. Chem. Soc. 101, 1913- 1937 (1979).

${ }^{50}$ I. M. Svishchev and P. G. Kusalik, "Dynamics in liquid $\mathrm{H}_{2} \mathrm{O}, \mathrm{D}_{2} \mathrm{O}$, and $\mathrm{T}_{2} \mathrm{O}$ : A comparative simulation study," J. Phys. Chem. 98, 728-733 (1994).

${ }^{51}$ In fitting, $C_{n}(t)$ it is assumed that $\mathrm{P}_{\alpha} A_{\alpha}=1$. As a consequence, in fitting $C_{n, H}(t)$ it is required that ${ }^{\mathrm{P}_{\alpha}}\left(\partial A_{\alpha} / \partial \beta\right)=0$.

${ }^{52 Z}$. A. Piskulich and W. H. Thompson, "On the temperature dependence of liquid structure," J. Chem. Phys. 152, 011102 (2020).

${ }^{53}$ R. C. Tolman, "Statistical mechanics applied to chemical kinetics," J. Am. Chem. Soc. 42, 2506-2528 (1920).

${ }^{54}$ D. G. Truhlar, "Interpretation of the activation energy," J. Chem. Educ. 55, 309-311 (1978).

${ }^{55}$ R. D. Levine and R. B. Bernstein, Molecular Reaction Dynamics and Chemical Reactivity (Oxford University Press, New York, 1987).

${ }^{56} \mathrm{~S}$. H. Northrup and J. T. Hynes, "The stable states picture of chemical reactions. I. Formulation for rate constants and initial condition effects," J. Chem. Phys. 73, 2700-2714 (1980).

${ }^{57}$ Previous work has typically considered only the long timescale of this decay, however, a global fit of the time correlation function is helpful in obtaining an accurate determination of the activation energies.

${ }^{58}$ S. Plimpton, "Fast parallel algorithms for short-range molecular dynamics," J. Comput. Phys. 117, 1-19 (1995).

${ }^{59} \mathrm{H}$. J. Berendsen, J. R. Grigera, and T. P. Straatsma, "The missing term in effective pair potentials," J. Phys. Chem 91, 6269- 6271 (1987).

${ }^{60} \mathrm{~T}$. Darden, D. York, and L. Pedersen, "Particle mesh Ewald: An N·log(N) method for Ewald sums in large systems," J. Chem.

Phys. 98, 10089-10092 (1993). 
${ }^{61 E}$ E. L. Pollock and J. Glosli, "Comments on PPPM, FMM, and the Ewald method for large periodic Coulombic systems," Comput. Phys. Comm. 95, 93-110 (1995).

${ }^{62}$ L. Martinez, R. Andrade, E. Birgin, and J. Martinez, "Packmol: A package for building initial configurations," J. Comput. Chem. 30, 2157-2164 (2009).

${ }^{63}$ S. Nos'e, "A unified formulation of the constant temperature molecular dynamics methods," J. Chem. Phys. 81, 511-519

(1984).

${ }^{64}$ W. G. Hoover, "Canonical dynamics: Equilibrium phase-space distributions," Phys. Rev. A 31, 1695-1697 (1985).

${ }^{65}$ D. P. Shoemaker, C. W. Garland, and J. W. Nibler, Experiments in Physical Chemistry (McGraw-Hill, New York, 1989).

${ }^{66} \mathrm{~F}$. Sciortino, A. Geiger, and H. E. Stanley, "Isochoric differential scattering functions in liquid water: The fifth neighbor as a network defect," Phys. Rev. Lett. 65, 5-8 (1990).

${ }^{67}$ L. Endom, H. G. Hertz, B. Thl, and M. D. Zeidler, "A microdynamic model of electrolyte solutions as derived from nuclear magnetic relaxation and self-diffusion data," Ber. Bunsenges Phys. Chem. 71, 1008-1031 (1967).

${ }^{68}$ A. A. Vartia, Reorientation and Solvation Dynamics of Bulk and Confined Alcohols, Ph.D. thesis, University of Kansas (2012).

${ }^{69} \mathrm{We}$ note that there may be some systematic error involved in our fits of the $n=1$ results for the frame reorientation as the fitted timescale is longer than the range of our data.

${ }^{70}$ D. E. Moilanen, D. Wong, D. E. Rosenfeld, E. E. Fenn, and M. D. Fayer, "Ion-water hydrogen-bond switching observed with 2D IR vibrational echo chemical exchange spectroscopy," Proc. Natl. Acad. Sci. 106, 375-380 (2009).

${ }^{71} \mathrm{M}$. Ji, M. Odelius, and K. J. Gaffney, "Large angular jump mechanism observed for hydrogen bond exchange in aqueous perchlorate solution," Science 328, 1003-1005 (2010). 